\title{
Is consciousness necessary for conflict adaptation? A state of the art
}

\author{
Kobe Desender ${ }^{1}$ and Eva Van den Bussche ${ }^{2 *}$ \\ ${ }^{1}$ Department of Experimental Psychology, Ghent University, Ghent, Belgium \\ ${ }^{2}$ Department of Psychology, Vrije Universiteit Brussel, Brussels, Belgium
}

\author{
Edited by: \\ Nicola De Pisapia, University of \\ Trento, Italy \\ Reviewed by: \\ Simon Van Gaal, University of \\ Amsterdam, Netherlands \\ Sachiko Kinoshita, Macquarie \\ University, Australia \\ *Correspondence: \\ Eva Van den Bussche, Faculty of \\ Psychology and Educational Sciences, \\ Vrije Universiteit Brussel, Pleinlaan 2, \\ 1050 Brussels, Belgium. \\ e-mail: eva.van.den.bussche@ \\ vub.ac.be
}

\begin{abstract}
Facing response conflict, subjects try to improve their responses by reducing the influence of the detrimental information which caused the conflict. It was speculated that this adaptation to conflict can only occur when the conflicting information is consciously perceived. In this review we give an overview of the research looking at the possibility of unconscious stimuli to provoke this conflict adaptation. In a first part we discuss adaptation to conflict on a trial-by-trial basis. When the previous trial contained conflicting information, subjects will adapt to this by reducing the influence of the conflicting information on the current trial. However, the interesting question is whether this is also possible when the conflicting information remains unconscious. In a second part we will discuss blockwise adaptation to conflict. If conflict is very frequent, subjects will adapt to this by reducing the conflicting information sustainably. Again the question is whether this is possible when the conflict was never experienced consciously. In a third part we will discuss the neural basis of conscious and unconscious conflict adaptation. We will critically discuss the research on these topics and highlight strengths and weaknesses of the used paradigms. Finally, we will give some suggestions how future research can be more conclusive in this respect.
\end{abstract}

Keywords: unconscious perception, conflict adaptation, cognitive control

\section{INTRODUCTION}

A hot topic in cognitive psychology nowadays is the question whether cognitive control can be exerted unconsciously. One typical expression of cognitive control is adaptation to response conflict. It has been convincingly shown that subjects adapt to conflicting information, by reducing the influence of this irrelevant information. The interesting question here is whether this is also possible when the conflict itself remains unconscious. Since much cognitive processing seems to be possible without any intervention of consciousness, this is an intriguing question. In this review we will give a clear overview of studies addressing this issue, and elaborate on contradictory findings in the field.

\section{THE POWER OF UNCONSCIOUS PROCESSING}

One of the most challenging questions in cognitive psychology is what defines the role of consciousness. As Lau (2009) points out, humans feel that without consciousness we would only be able to perform simple, reflexive behavior. Intuitively, we claim that we need consciousness to execute complex behavior based on our conscious intentions. Most people would agree that consciousness has an important function in life, although it is difficult to spell out what it is precisely. However, the answers from science are not unequivocal. An overwhelming body of research has shown that many cognitive processes can occur without consciousness meddling in. Yet this consensus is a very recent one. It has long been a spirited topic of debate whether stimuli which do not pass the consciousness threshold can be processed at all. Skeptics claimed that almost all research failed to assure that stimuli were truly unconscious (e.g.,
Eriksen, 1960; Holender, 1986) and false claims about subliminal advertising did more harm than good (Pratkanis, 1992). But with methodological improvements, such as better masking paradigms (Evett and Humphreys, 1981; Forster and Davis, 1984) and objective prime awareness tests (Greenwald et al., 1996) to ensure the unconscious nature of the stimuli, the consensus grew that stimuli which never enter our consciousness are nevertheless capable of influencing on going behavior (Kouider and Dehaene, 2007). Not only is the existence of unconscious perception no longer questioned, accumulating evidence has highlighted the potentially far-reaching power of unconscious processing.

\section{Unconscious semantic activation}

Much research on unconscious processing made use of the priming paradigm. In this paradigm subjects have to respond to a target which is preceded by an irrelevant prime. Responses to this target are faster when it is preceded by a prime which triggers the same response as the target (i.e., a congruent prime) compared to a prime which triggers a different response (i.e., an incongruent prime). This congruency effect is a robust phenomenon both when the prime is clearly visible and when it is masked (e.g., Vorberg et al., 2003). Initially, it was assumed that masked, invisible stimuli could only trigger automatic response priming. For example, Eimer and Schlaghecken (1998) showed that a prime arrow, which is displayed for only $16 \mathrm{~ms}$, is capable of activating motor responses. Dehaene et al. (1998) went one step further and found that a subliminally presented prime number facilitated responses to a target number when they shared a semantic 
relation, suggesting that unconscious stimuli can be processed up to a semantic level.

Although this semantic interpretation was not generally accepted at first (Damian, 2001; Kunde et al., 2003), there have been convincing studies which showed that a subliminal prime is indeed capable of influencing the reaction to a target via their semantic relation (Van den Bussche et al., 2009a; Van Opstal et al., 2010). A meta-analysis on masked priming studies (Van den Bussche et al., 2009b) confirmed that even when all non-semantic influences are excluded, subliminal primes can still evoke priming effects. So, unconscious primes can reach a high semantic processing level. Following the explanation by Dehaene et al. (1998) we assume that these masked priming effects are the consequence of conflict at the response level. On congruent trials, the prime activates the same response as the target, whereas on incongruent trials both sources active a different response, and this response conflict slows down responding (for a different view see Kinoshita and Hunt, 2008).

\section{Top-down influences on unconscious processing}

Next to being able to reach semantic processing levels, the effect of subliminal stimuli is not restricted to purely bottom-up processing. Although the influence of unconscious primes has often been thought a static, uncontrollable process, recently research pointed out that it can be influenced by top-down aspects such as task demands (Norris and Kinoshita, 2008; Martens et al., 2011), attentional focusing (Van den Bussche et al., 2010), and temporal attention (Naccache et al., 2002). Martens et al. (2011) used an induction task to activate one of two task sets. Subjects had to decide whether pictures of objects where living or non-living (i.e., a semantic induction task) or whether they were round or elongated (i.e., a perceptual induction task). This induction task had an influence on a subsequent priming task. After the semantic induction task priming was only observed when prime and target shared a semantic relationship, but not when they only shared a perceptual relationship. After the perceptual induction task the opposite was true. Likewise, Norris and Kinoshita (2008) showed in a masked priming task that the priming effect for non-words was dependent on the task. In a standard lexical decision task, no unconscious priming for non-words was observed. However, when exactly the same prime-target pairs were used, but now the task was to decide whether the target was the same as a probe, unconscious priming was also observed for non-words. Van den Bussche et al. (2010) showed that spatial attention is a prerequisite for subliminal stimuli to be processed. When attention was allocated to another location, primes had no influence on the processing of the target. In a similar vein, Naccache et al. (2002) demonstrated that subliminal primes only influenced responses to targets when temporal attention was allocated to the time window in which the prime appeared. When this was not the case, no priming effects emerged.

These studies illustrate that subliminal priming is not an inflexible process which operates in a purely automatic, bottom-up fashion. Our conscious preparation can have large modulating effects on the processing of unconscious primes (e.g., Kunde et al., 2003).

\section{THE LIMITS OF UNCONSCIOUS PROCESSING}

As described above, accumulating evidence shows that unconscious stimuli can be processed upto a high semantic level, and that it is susceptible to several conscious top-down modulations. Over the years the consensus grew that many mental activities can be performed without awareness, and the intriguing question arose which processes require consciousness. Does unconscious processing have limits and does consciousness have a special function at all? A controversial position on this topic was taken by Libet (1985) who speculated that consciousness and free will are illusory (see also Wegner, 2002). He showed that the brain activation of an action is present in the electroencephalogram (EEG) waveform, several hundreds of milliseconds before the subject has the conscious experience of initiating the action. Recently this finding was extended by Soon et al. (2008) who showed that the decision to perform an action was measurable in prefrontal and parietal cortex, up to $7 \mathrm{~s}$ before subjects consciously decided to act. So it seems that consciousness has nothing to do with initiating actions, but subjects only post hoc create the illusion that they deliberately produced the action.

Contrary to this extreme position, Dehaene and Naccache (2001) argued that there are borders which determine the potential role of unconscious processing. They proposed a framework in which specialized modular systems can process stimuli without conscious amplification, as long as this is merely a bottom-up process which requires no strategic adaptation. This bottom-up processing of unconscious stimuli can be altered by the processing state of our cognitive system, such as the focus of attention or the currently activated task set, as long as this is initiated consciously. What should, however, not be possible, is for an unconscious stimulus itself to change these top-down strategies of participants. Unconscious processing can only use these purely bottom-up modular systems in the brain. According to Dehaene and Naccache (2001), without global ignition (which is the determinant of consciousness) these stimuli cannot initiate top-down cognitive control, because they remain within a modular system. According to this proposal, consciousness should be exclusively associated with strategic operations such as planning a new strategy, evaluating it, controlling its execution and correcting possible errors. All these operations can be grouped under the term cognitive control.

If we want to test whether consciousness is more than just an epiphenomenon without any purpose, it is necessary to capture those behaviors which cannot be initiated by unconscious stimuli. Focusing on cognitive control operations might offer a fruitful approach to explore this debated issue, since they have been specifically associated with consciousness (Dehaene and Naccache, 2001). This is of course not an easy task to accomplish, because we are looking for the absence of an effect, and this can almost always be explained by alternative interpretations. Thus we need convincing research which is completely free from methodological flaws, to be able to answer this question.

\section{COGNITIVE CONTROL AND CONFLICT ADAPTATION}

Because cognitive control is not a well-defined area of human actions, it is important to specify the behavior under investigation. 
Many different behaviors are categorized under the term cognitive control, and most definitions are very broad. For example, Botvinick et al. (2001) defined cognitive control as the human cognitive system's "ability to configure itself for the performance of specific tasks through appropriate adjustments in perceptual selection, response biasing, and the on-line maintenance of contextual information" (p. 624). Although exhaustive, we risk that with such a general definition, in the end, almost all behavior is an instance of cognitive control. Therefore in this review we will focus on one commonly investigated expression of cognitive control, namely the ability to detect response conflict and adjust our behavior accordingly. It should be stressed that research on unconscious processing was also carried out in other domains of cognitive control. Typically, it is found that instances of cognitive control can be influenced unconsciously, if subjects are familiarized with the conscious variant of the task before. For example, if subjects have learned to withhold a response when a visible stop-signal is presented, responses will also slow down when this stop-signal is presented unconsciously (e.g., van Gaal et al., 2010a,c). Likewise, if subjects have learned which task to perform dependent on a task cue, the task can be primed by presenting the corresponding cue unconsciously (Lau and Passingham, 2007; De Pisapia et al., in press; Reuss et al., 2011). Finally, post-error slowing can occur, even when subjects are completely unaware of making an error (Hester et al., 2005; Cohen et al., 2009). Thus, although detecting response conflict and adjusting our behavior accordingly is a commonly investigated expression of cognitive control, others also exist.

A typical example of conflict adaptation can be found in the Stroop task, where subjects are confronted with color words (e.g., green) which are printed in a color that can either match the word meaning (e.g., green; a congruent situation) or not match the word meaning (e.g., red; an incongruent situation). Subjects are asked to name the ink color (i.e., the relevant information) as fast as possible, while ignoring the word meaning (i.e., the irrelevant information). In an incongruent situation both sources of information trigger differential responses, and thus create response conflict, which slows down responding. Consequently, responses are usually much faster in the congruent condition than in the incongruent condition (i.e., the congruency effect). This response conflict can occur in all tasks in which relevant and irrelevant information can potentially activate differential responses. To prevent this irrelevant information to exert the same detrimental influence later on, our cognitive system will adapt to this conflict so that in a subsequent conflict situation we are better in dealing with the conflict, and thus in reducing its detrimental influence. It was proposed that the cognitive system adapts to this response conflict by inhibiting the irrelevant information (Stürmer et al., 2002) or by narrowing attention to the relevant information (Egner and Hirsch, 2005). Wühr and Frings (2008) showed in a within-trial paradigm that target amplification and distractor suppression presumably interact in selecting the correct answer. Because the precise mechanism of conflict adaptation remains debated, we prefer to remain theoretically neutral when using this term. Throughout this review, we will use the term conflict adaptation to refer to the behavioral effects following response conflict. Likewise, although other kinds of conflict exist, our focus lies on response conflict. Thus, when using the term conflict we are referring to response conflict.

We can dissociate two strategies to cope with this kind of response conflict. We can handle the conflict on a trial-by-trial basis and adjust our response strategy according to the conflict in the current trial (i.e., micro-adjustments; Ridderinkhof, 2002). Alternatively we can pick up regularities over a longer period of time and adjust our strategy blockwise, based on this accumulated information (i.e., macro-adjustments; Ridderinkhof, 2002).

The next section is divided into two parts in which we will discuss these trial-by-trial and blockwise strategies separately. After a brief overview of the literature we will elaborate on experiments investigating the (im)possibility of unconscious conflict to cause these kinds of conflict adaptation.

\section{AN AREA EXCLUSIVELY RESERVED FOR CONSCIOUSNESS? CONFLICT ADAPTATION ON A TRIAL-BY-TRIAL BASIS}

The strategy to cope with response conflict on a trial-by-trial basis was explored by Gratton et al. (1992) with the Eriksen flanker task (Eriksen and Eriksen, 1974). In this task subjects have to respond to a central target letter (A or $\mathrm{H})$. This target letter is flanked by irrelevant distractor letters. These distractors can trigger the same response as the target letter (i.e., a congruent situation; AAAAA) or they can trigger the opposite response (i.e., an incongruent situation; HHAHH). Although the distractor letters are completely irrelevant for the task, they nevertheless exert a great influence; large congruency effects are observed under these conditions. Interestingly, the authors observed that the congruency effect was modulated by the congruency on the previous trial. If there was conflict (i.e., an incongruent trial) on the previous trial, they observed a smaller congruency effect on the current trial, compared to when the previous trial was congruent. This implies that subjects, facing conflict, adapt to this conflict by reducing the source of the conflict (the irrelevant information) on the following trial. Thus although subjects are not able to simply ignore the irrelevant information throughout the whole task, they nevertheless adapt their behavior on a trial-by-trial basis based on this irrelevant information.

These sequential modulations have also been shown in other paradigms such as the Stroop task (Kerns et al., 2004; Notebaert et al., 2006; Lamers and Roelofs, 2011), the Simon task (Stürmer et al., 2002; Akçay and Hazeltine, 2008; Verguts et al., 2011), and the priming paradigm (Kunde, 2003; Kunde and Wühr, 2006). Apparently it does not matter whether conflict is conveyed by an irrelevant flanker, an irrelevant word meaning, an irrelevant position, or an irrelevant arrow (for a different view, see Egner, 2008). Whenever the cognitive system detects conflict it will adapt to it and reduce the influence of the irrelevant information on the next trial.

\section{UNCONSCIOUS CONFLICT ADAPTATION ON A TRIAL-BY-TRIAL BASIS}

The previous part made it clear that subjects cope with conflict on a trial-by-trial basis by reducing the influence of the irrelevant information on the following trial. The interesting question now is whether this adaptation also occurs when the conflict itself remains unconscious (see Table $\mathbf{1}$ for an overview of studies addressing this 
Table 1 | Schematic overview of studies looking at adaptation to conflict on a trial-by-trial basis.

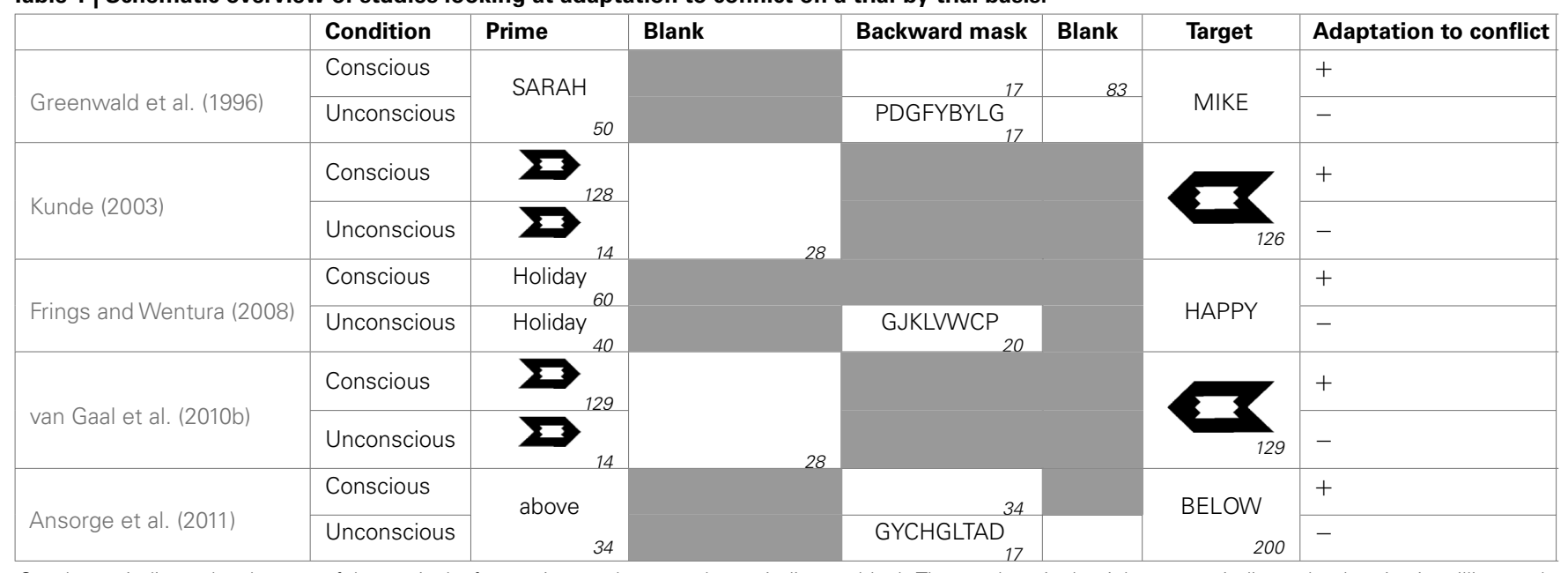

Gray boxes indicate the absence of the particular feature in a study, empty boxes indicate a blank. The numbers in the right corners indicate the duration in milliseconds.

issue). To answer this question, Kunde (2003) used a meta-contrast masking paradigm. In this paradigm a prime arrow fits perfectly in the target contour, so that this first arrow is rendered invisible if both are presented in short succession. To create conscious and unconscious conditions, the prime duration was randomly varied between 14 and $126 \mathrm{~ms}$, followed by a blank of $28 \mathrm{~ms}$. Independent of the prime duration on the current trial, Kunde found a reduction of the congruency effect only when a $126 \mathrm{~ms}$ prime was presented on the previous trial (similar results were presented by Greenwald et al., 1996). Based on this observation, he concluded that we can only adapt to conflict on a trial-by-trial basis when this conflict is consciously experienced. An important problem with this study is that the prime duration differed between the conscious and unconscious conditions. This changed the time between the onset of the prime and the onset of the target stimulus onset asynchrony (SOA) which is sufficient to influence priming effects (Eimer and Schlaghecken, 1998).

In an affective priming experiment Frings and Wentura (2008) held the SOA fixed at $60 \mathrm{~ms}$. On every trial they presented a prime word with a positive or negative valence, followed by a target word which could be either congruent (i.e., the same valence) or incongruent (i.e., the other valence) with the prime. In the conscious condition the prime was presented $60 \mathrm{~ms}$, without a mask. In the unconscious condition the prime was only presented $40 \mathrm{~ms}$ followed by a 20 ms post-mask. Replicating Kunde (2003) they found a sequential modulation of the congruency effect on the current trial only when an unmasked prime was presented on the previous trial.

Contrary to the these studies, which indicate the impossibility of unconscious conflict to alter the processing of the next trial, van Gaal et al. (2010b) challenged the hypothesis that conflict which remains unconscious can never recruit top-down cognitive control. They used the same experiment as Kunde (2003), but omitted a brief auditory warning signal at the beginning of each trial and shortened the inter-trial interval. Contrary to Kunde (2003) they observed conflict adaptation on the current trial, independent of the visibility of either the current or the previous trial. According to the authors, the auditory signal in the study of Kunde
(2003) disturbed subjects' attention and thereby wiped out any unconscious traces. Two recent studies also claimed to have found adaptation to unconscious conflict. In a priming study, Francken et al. (2011) obtained conflict adaptation on the current trial, independent of the visibility of the previous trial. However, this was only the case in the error rates, not in reaction times. Moreover, the visibility of their low-visibility condition was too high to be considered unconscious. Bodner and Mulji (2010) presented only masked primes, and manipulated conflict frequency. For half of the participants $80 \%$ of the trials were congruent, for the other half $80 \%$ of the trials were incongruent. A larger priming effect for the group receiving $80 \%$ congruent trials was only apparent when the previous trial was incongruent or neutral. When the previous trial was positive, no different priming effects were observed. Although this shows that congruency effects are dependent on the congruency of the previous trial, these results are qualitatively different from those presented by van Gaal et al. In the group receiving $80 \%$ incongruent trials, Bodner and Mulji (2010) observed numerically smaller congruency effects following incongruent trials. However, in the group receiving $80 \%$ congruent trials, the congruency effect was numerically larger following an incongruent, compared to a congruent trial. This observation is unexpected, and it is currently unclear how this can be reconciled with the findings of van Gaal et al.

\section{ALTERNATIVE EXPLANATIONS}

Based on the studies of van Gaal et al. (2010b) and Francken et al. (2011) one could conclude that unconscious conflict can trigger adaptation behavior. An important question is whether these results are truly the consequence of unconscious conflict, or alternatively the consequence of information which reached consciousness (e.g., Ansorge et al., 2011). For example it is possible that van Gaal et al. (2010b) found unconscious conflict adaptation, not because the conflict exerted an influence on behavior unconsciously, but because participants became somehow aware of the conflict on a meta-cognitive level (e.g., Van den Bussche et al., 2008). For example, responses are typically slower on incongruent trials, and perhaps subjects became consciously aware 
of this reaction time slowing. It has been shown that subjects can reliably "read out" their own reaction times in a conscious manner (Corallo et al., 2008; Marti et al., 2010). Perhaps adaptation on the current trial can be triggered by the conscious experience that we somehow responded slower on the previous trial. Another way in which we can consciously experience differences on the previous trial is because of different senses of control. Recently, in a masked priming study it was shown that our sense of control over action effects (i.e., a stimulus presented after responding) is larger when the effects are produced by congruent compared to incongruent trials (Wenke et al., 2010). Thus, although subjects are never consciously aware of any prime, they nevertheless feel more in control over the effects of congruent compared to incongruent trials. A similar possibility is that subjects consciously experienced that responding was somehow easier on congruent than on incongruent trials, although the reason for this experience remained unconscious. In this case, subjects adapted their behavior on the current trial, based on the consciously experienced difficulty of the previous trial, rather than the unconscious conflict of the previous trial (e.g., Kinoshita et al., 2011).

This last point was also noted by Ansorge et al. (2011). In their priming experiment, all primes were followed by a $34 \mathrm{~ms}$ blank or a $34 \mathrm{~ms}$ mask, to create conscious and unconscious conditions. To control for meta-cognitive explanations of sequential modulations, they collected visibility data on a trial-by-trial basis. After every trial, participants were asked whether they had just encountered a congruent or an incongruent trial. When the authors analyzed only those trials on which subjects wrongly judged the congruency of the previous trial, no sequential modulations were found at all. This suggests that even in the unmasked condition conflict awareness plays a major role. When they analyzed only trials in which subjects correctly identified the congruency of the previous trial, they observed sequential modulations dependent on the congruency of the previous trial, only when the conflicting information on the previous trial was unmasked. This suggests that unconscious trial-by-trial adaptations cannot be caused by subjects becoming aware of the conflict on some level. However, also this conclusion is premature. The main advantage of this trialby-trial assessment is that we can determine whether unconscious trial-by-trial adaptations can be caused by responses on those trials where subjects, despite the masking, were consciously aware of the prime or the prime-target congruency on the previous trial. Importantly, these trials should be distinguished from trials on which subjects really had no idea about the congruency at all, because in the latter case an unconscious sequential modulation effect cannot be explained by meta-cognitive processes. Unfortunately Ansorge et al. (2011) gave participants only two response options in the visibility question ("was the trial you just saw congruent or incongruent?”). In this way, a confound exists between genuine judgments and instances in which subjects really had no idea and just guessed. This can conceal a possible unconscious conflict adaptation effect.

\section{CONCLUSION}

In summary, although van Gaal et al. (2010b) and Francken et al. (2011) recently observed adaptation to conflict on a trial-by-trial basis, even when the conflicting information on the previous trial was masked, it would be premature to conclude that sequential modulations can be initiated by unconscious conflict. Research on sequential modulations has repeatedly shown that subjects only adapt to conflict on the previous trial, when this conflict was experienced consciously. Furthermore, an alternative metacognitive explanation can be readily offered for the findings of van Gaal et al. In the Section "General Discussion" we will discuss the conditions required to investigate this unresolved issue further.

\section{AN AREA EXCLUSIVELY RESERVED FOR CONSCIOUSNESS? BLOCKWISE ADAPTATION TO RESPONSE CONFLICT}

The second strategy described by Ridderinkhof (2002; macroadjustments) to adapt to conflict is based on detecting blockwise regularities of conflict trials. This is a useful strategy when, for example, congruency proportions are manipulated. If in a block the proportion of incongruent trials is much larger than the proportion of congruent trials, the level of conflict is generally elevated. Since in this situation most trials contain conflict, the most economical strategy is probably to simply reduce the detrimental influence of the irrelevant information continuously. This strategy is adequate for most trials and thus seems an acceptable way to deal with the conflict. Adapting our behavior on a trial-bytrial basis according to the conflict on the previous trial is also a potential strategy here, but this would be much more cognitively demanding because the majority of trials contain response conflict and the cognitive system would have to reduce the influence of the irrelevant information repeatedly. Instead, it would be much easier for the cognitive system to simply reduce the influence of the irrelevant information sustainably. There is much evidence that subjects effectively deal with these blockwise statistical regularities. For example Logan and Zbrodoff (1979) used a Simon task and varied the congruency proportions. When $90 \%$ of the trials were congruent, subjects showed a large congruency effect, with faster reactions on congruent compared to incongruent trials. On the other hand when $90 \%$ of the trials were incongruent, this pattern was reversed. Subjects now showed an inversed congruency effect, with faster reactions on incongruent compared to congruent trials. This seems to imply that subjects somehow kept track of the ratio of incongruent vs. congruent trials, and used this information to improve their responses. This blockwise adaptation to response conflict is also observed in other paradigms such as the Stroop task (Tzelgov et al., 1992; Merikle and Joordens, 1997), the Eriksen flanker task (Gratton et al., 1992; Purman et al., 2011), and the priming paradigm (Klapp, 2007; Jaskowski, 2008). So as with trial-by-trial adaptation, it does not seem to matter how conflict is conveyed. As long as there are regularities in a block, subjects will adapt to this manipulation by changing the impact of the irrelevant information on responding.

\section{BLOCKWISE ADAPTATION TO UNCONSCIOUS RESPONSE CONFLICT}

Based on the previous part we know that subjects strategically adapt blockwise to the frequency of conflict trials in the experiment. Are we also able to do this, when the conflicting information remains unconscious (see Table 2 for an overview of studies addressing this issue)? Merikle and Joordens (1997) addressed this issue in an adjusted Stroop paradigm. Their subjects had to name the ink color of an array of colored ampersands. These were 
Table 2 | Schematic overview of studies looking at blockwise adaptation to response conflict.

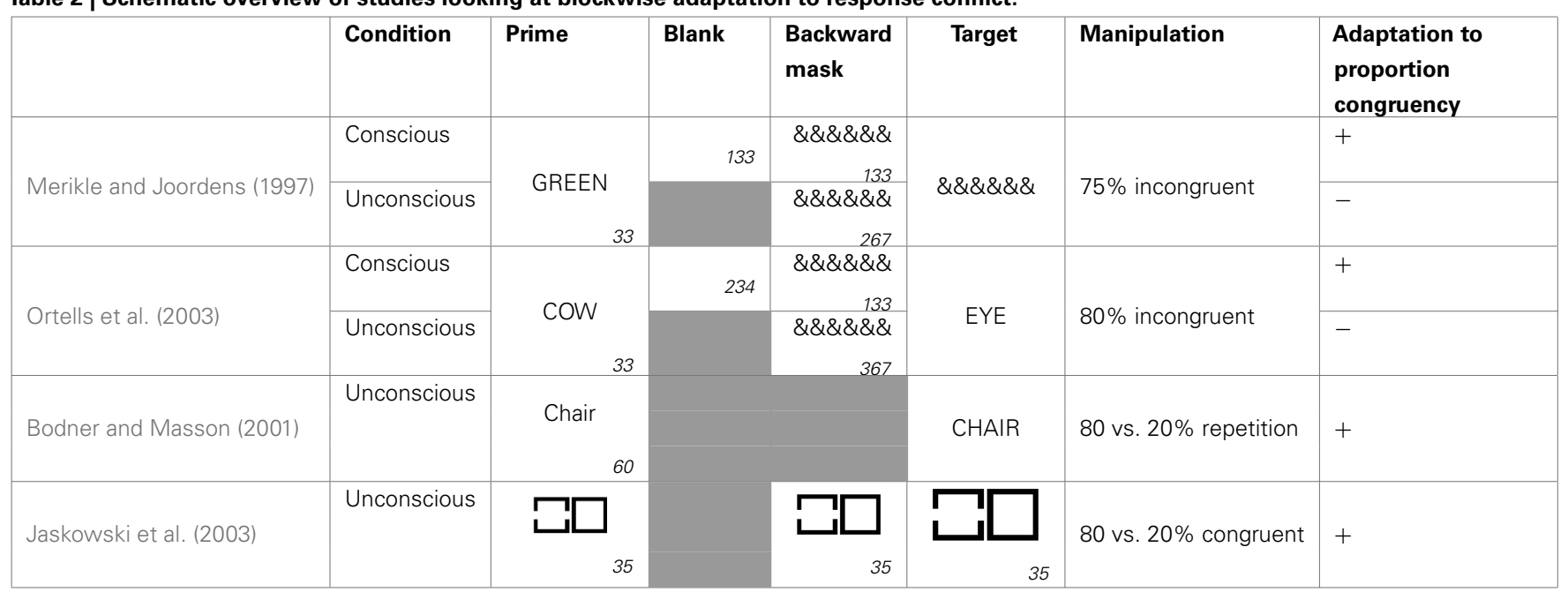

Gray boxes indicate the absence of the particular feature in a study, empty boxes indicate a blank. The numbers in the right corners indicate the duration in milliseconds.

preceded by a color word presented in gray which was incongruent in $75 \%$ of the cases with the response to the ampersands. Only in $25 \%$ of the trials the color word triggered the same response as the ampersands. Under these conditions subjects were able to strategically predict the color of the ampersands based on the color word, leading toan inversed congruency effect. The interesting observation was that subjects were only able to strategically use this conflict frequency information, when the color word was presented visibly. When this was masked, a normal Stroop effect occurred (see also Daza et al., 2002).

Similar results were presented by Ortells et al. (2003). They used a semantic priming task in which participants had to classify target words as denoting animals or body parts. In $80 \%$ of the trials the targets were preceded by a prime word of a different category (e.g., HAND-dog) and only in $20 \%$ by a semantically related prime (e.g., HAND-finger). When the primes were presented visibly, subjects were able to make use of this information leading to an inversed congruency effect. But again, if the primes were masked, subjects showed a normal congruency effect. This effect appeared to be very robust, emerging when masked and unmasked trials were randomly mixed within the same experiment (Ortells et al., 2006) and when a different task had to be performed on the targets (Daza et al., 2007).

Although these experiments highlight a qualitative difference between conscious and unconscious conditions, it cannot be assured that there was completely no blockwise adaptation to the unconscious frequency manipulation. Since these studies did not provide a baseline measure of the congruency effect, we cannot ascertain whether the congruency effect in the masked condition was, although not reversed, nevertheless diminished. Other authors, who did include a baseline condition, found blockwise adaptation to unconscious response conflict with comparable paradigms. For example, Bodner and Masson (2001) used a lexical decision task in which participants had to decide as quickly as possible whether a target string printed in uppercase (e.g., CHAIR) was a word or a nonsense letter string. Unknown to the participants the target was preceded by a $60 \mathrm{~ms}$ prime word in lower case. This could be a repetition of the target (e.g., chair) or an unrelated word. The authors compared groups receiving blocks where $80 \%$ of the primes were repetitions of the target and groups where only $20 \%$ of the primes were repetitions, and thus $80 \%$ of the primes were neutral. They found that participants adapted to these regularities, and the priming effect was magnified in blocks containing $80 \%$ repetition primes. This finding was repeatedly replicated and extended (see for example Bodner and Masson, 2004; Bodner and Dypvik, 2005; Bodner et al., 2006; Klapp, 2007; Bodner and Mulji, 2010).

A major problem with the previously cited studies is the prime visibility. If primes in these studies were not truly unconscious, we cannot exclude the possibility that these effects are driven by conscious influences. For example in Bodner and Masson (2001), although $77 \%$ of the subjects did not notice the primes, we cannot simply assume based on this information that their primes were truly unconscious. Additional measures need to be gathered to ensure that the results are not caused by subtle conscious influences. Unfortunately, all mentioned studies fail to satisfy this criterion. Often no objective prime awareness data is available (Bodner and Masson, 2001, 2004; Bodner et al., 2006), or subjects classify primes above chance (Klapp, 2007) and authors fail to report necessary statistics such as a significant intercept, showing priming at visibility zero (Bodner and Dypvik, 2005).

Interestingly, Jaskowski et al. (2003) showed blockwise adaptation to unconscious frequency manipulations, with a perfectly masked prime. In a priming experiment participants were presented with two target squares side by side, and had to decide as quickly as possible which of the two contained a gap. Unknown to participants, each target square was preceded by four other squares, which were shortly flashed in short succession. One or more of the first four squares also had a gap and served as primes for the target square. Each square fitted perfectly in the following, so that gaps in the prime were rendered invisible. The gaps could appear in a prime flashing before the target with a gap (i.e., congruent) or before the target without a gap (i.e., incongruent). They observed a strategic adaptation dependent on the ratio of congruent vs. incongruent trials. When $80 \%$ of the trials where congruent 
they observed a strong congruency effect $(130 \mathrm{~ms})$. When, on the contrary, only $20 \%$ of the trials were congruent they observed a reduction of the congruency effect $(55 \mathrm{~ms})$.

\section{ALTERNATIVE EXPLANATIONS}

Although the study of Jaskowski et al. (2003) seems to provide evidence that subjects adapted their behavior based on unconscious conflict, the authors instead explained their results by assuming a meta-cognitive process. They proposed that subjects are not aware of the conflict per se, but they became aware of a consequence of unconscious conflict, namely error tendencies. Because in blocks with $80 \%$ incongruent trials the prime mostly signals the erroneous response, errors are much more likely in this situation. Subjects might become aware of a higher tendency to commit errors in blocks containing mainly incongruent trials, and thereby strategically adjust their behavior to a more conservative response approach. This interpretation of their results fits nicely with the hypothesis that an unconscious stimulus itself is not able to recruit cognitive control to change on going behavior, but the conscious experience of a result of this unconscious stimulus is (Dehaene and Naccache, 2001). A similar argument was made by Van den Bussche et al. (2008). In a priming study they found a larger congruency effect for the target notation (e.g., Arabic number vs. number word) which was also presented as prime in $75 \%$ of the cases. They explained this unexpected result by suggesting that subjects may become aware of the facilitation of the target format which is also presented in $75 \%$ of the primes, without being aware of the prime or the relation between prime and target. Because of the awareness that these targets are somehow easier to respond to, subjects focus more on them, which results in stronger priming effects. These observations suggest that the effects of unconscious manipulations can be very subtle and indirect, while at first glance they seem to provide evidence for unconscious information being used to adapt subsequent behavior.

Kinoshita et al. (2008) proposed an explanation along similar lines in their Adaptation to Statistics of the Environment (ASE) model. They assume that subjects' response strategy, according to a speed accuracy dimension, is based on the difficulty of the task at hand. If an experiment contains mainly congruent primes, the task is very easy, because the prime always facilitates responding to the target. Therefore subjects can try to respond very fast without the risk of making too many errors. This will shorten reaction times to these easy targets and thus prolong reactions on the infrequent difficult (incongruent) trials. If an experiment contains mainly incongruent trials, the task at hand is now much harder. Because the prime always signals the wrong response, subjects will adopt a more cautious response deadline, in order to prevent making too many errors. If the proportion of congruent and incongruent trials is equal, a response deadline in between these two extremes will be appropriate. This will slow down responses to congruent trials and speed up responses to incongruent trials. Kinoshita et al. (2011) argued that this response deadline is mainly influenced by the difficulty of the previous trial. If the previous trial was difficult we will adopt a more cautious response strategy compared to when the previous trial was easy. Moreover, these effects are asymmetric. That is, the response to an easy trial is more sensitive to the difficulty of the previous trial than the response to a difficult trial. In their experiment 3 , the authors showed that the blockwise adaptation to unconscious frequency manipulations can in fact be explained by previous trial difficulty. According to Kinoshita et al. (2011), the ASE-model can explain all studies claiming to have found blockwise adaptation to unconscious response conflict. It can be argued that the ASE-account can be seen as a more general extension of the proposal by Jaskowski et al. (2003). In the ASE-model not only the conscious error rate, but task difficulty in general can influence responses. In order for task difficulty to influence response strategies, subjects must on some level become aware of this difficulty, probably without knowing the specific origin (Van den Bussche and Reynvoet, 2008). A possible explanation for this awareness is the conscious "read out" of reaction times (Corallo et al., 2008; Marti et al., 2010). Subjects may become aware of the difficulty of the previous trial, because they consciously notice that responses on difficult (i.e., incongruent) trials are slower than on easy trials.

To summarize, the meta-cognitive accounts discussed here provide a viable alternative explanation for observed results, and point to an important methodological drawback in current research. According to the ASE-model, the most important problem when manipulating conflict frequency blockwise is that task difficulty and congruency proportion are always confounded. As a consequence, an alternative explanation can always be that subjects somehow consciously experience the task difficulty (Van den Bussche and Reynvoet, 2008) or the error tendency (Jaskowski et al., 2003). Therefore alternative ways should be addressed to investigate this question. The most important problem which has to be dealt with is the possibility that statistical regularities can somehow reach consciousness.

\section{CONTEXT EFFECTS}

A possible solution to disentangle congruency and task difficulty is to create one context with mainly congruent trials and one with mainly in congruent trials, and randomly switch between them within the same block. Thus, on each trial the probability of each context is perfectly equal. However, the probability to encounter a congruent trial is highly dependent on the context. Because every trial is randomly of either the mainly congruent or the mainly incongruent type, the various difficulties of both contexts (Kinoshita et al., 2008) cancel each other out (see Table 3 for an overview of studies addressing this issue). Because participants need to be able to dissociate between both contexts, they need to have a way to know which of both is at hand. This is exactly what Heinemann et al. (2009) did. In a priming study, subjects had to decide whether a target digit was larger or smaller than five. Every trial started with a fixation cross, followed by a prime digit lasting $26 \mathrm{~ms}$, a blank (60 ms), a mask ( $10 \mathrm{~ms})$, and the target digit. Along with the fixation cross the authors presented a context cue in the form of a colored rectangle, whose color indicated with $80 \%$ certainty whether the upcoming trial would be congruent or incongruent. As expected, they observed that the congruency effect was larger in the context with $80 \%$ congruent trials compared to the context with $80 \%$ incongruent trials. This context-specific prime-congruency effect (i.e., CSPC) was shown before (e.g., Crump et al., 2006; Crump and Milliken, 2009), but Heinemann et al. found that it only occurred when the prime was 
Table 3 | Schematic overview of studies looking at the possibility of unconscious context effects.

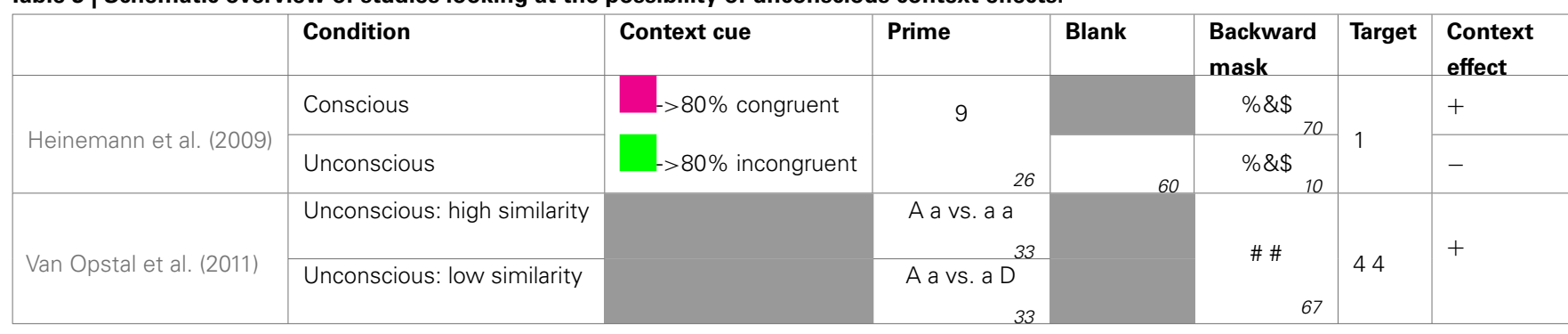

Gray boxes indicate the absence of the particular feature in a study, empty boxes indicate a blank. The numbers in the right corners indicate the duration in milliseconds.

truly visible. When they filled the 60 ms blank with a mask, which rendered the prime unconscious, the congruency effect did no longer differ between the two contexts. Although the context cue was still clearly visible, its moderating effect completely vanished. Apparently, subjects in the masked condition never acquired the contingency between the cue and the congruency of the upcoming trial. A possible explanation is that an arbitrarily chosen cue is not significant enough for participants to be automatically linked to statistical regularities which remain unconscious (Van Opstal et al., 2011). To examine this possibility, future studies need to create more task-relevant, self-evident contexts, compared to presenting an arbitrary cue before every trial.

Van Opstal et al. (2011) took a first step in this direction by showing that the influence of primes on targets can be reversed by an unconscious context. In their experiment subjects had to decide whether two target digits (e.g., 4 4) were the same or different. Unknown to participants, the targets where preceded by two prime letters, which also could be equal or not. The authors compared the priming effect of moderate similar primes (e.g., a A), dependent on the context in which they were presented. Half of the subjects received these unconscious primes in a context of completely dissimilar primes (e.g., a D). In this situation these moderate similar primes were relatively equal and as a consequence facilitated "same" responses. Conversely, the other half of the subjects received these unconscious primes in a context of completely similar primes (e.g., a a). In this situation these moderate similar primes were relatively different, and as a consequence facilitated "different" responses. This nicely illustrates that an unconscious context can affect our behavior.

\section{CONCLUSION}

Summarizing research on blockwise adaptation to unconscious response conflict, the emerging picture is complicated. In an attempt to disentangle conscious and unconscious processes, some studies showed inversed congruency effects, as an adaptation to blockwise manipulation of conflict frequency, for conscious conditions only (e.g., Merikle and Joordens, 1997; Daza et al., 2002). They interpret these qualitative differences as evidence that cognitive control is exclusively reserved for consciousness. Studies showing blockwise adaptation to conflict frequency, even in unconscious conditions, challenge this conclusion (e.g., Bodner and Masson, 2001, 2004; Klapp, 2007). Nevertheless, no study to date has shown reversed congruency effects when the conflict itself remained unconscious. So a tempting conclusion would be that there are graded differences between adaptation to conscious and unconscious conflict, but adaptation can occur in both situations. Unfortunately, this conclusion is premature, since alternative meta-cognitive accounts have challenged the notion of blockwise adaptation to unconscious response conflict (e.g., Jaskowski et al., 2003; Kinoshita et al., 2008, 2011). Currently, there is just a single study to counter these criticisms (Van Opstal et al., 2011), so it would be worthwhile to test its robustness and generality in future research.

\section{CONFLICT ADAPTATION: THE NEURAL BASIS}

Behavioral studies on conflict adaptation showed a large discrepancy in the literature. Many studies find robust effects of adaptation to response conflict, when the conflict is consciously perceived. When conflict remains unconscious, the evidence is far from clear-cut. A possible tool to answer this question is to broaden the scope of research methods, and look at the neural basis of conflict adaptation. Using functional MRI (fMRI) and EEG, we can study whether certain brain areas are differentially activated by conscious and unconscious conflicting information.

\section{CONFLICT ADAPTATION IN THE BRAIN}

An interesting question is how the brain knows when to reduce the influence of conflicting information. In their conflict monitoring theory, Botvinick et al. (2001) proposed that the anterior cingulate cortex (ACC) constantly monitors for response conflict. They presume that when differential responses are suggested by stimuli, this activates the ACC which detects this response conflict. Subsequently it will in turn activate the prefrontal cortex (PFC), which then takes remedial actions to reduce this conflict. A critical discussion of this proposal lies beyond the scope of this review, so we will confine us to a clear overview of brain studies addressing response conflict. In the Stroop paradigm, there is much evidence that the ACC is indeed activated when the word meaning and the ink color trigger a different response (MacLeod and MacDonald, 2000). Also in the flanker and the Simon task, the ACC proved to be activated when there was conflict between the relevant and the irrelevant stimulus dimension (Botvinick et al., 2004; Botvinick, 2007). There is also evidence for the hypothesis that, when facing conflict, the ACC activates the PFC to cope with this conflict. In an fMRI study using the Stroop task, Kerns et al. (2004) observed that the congruency effect on the current trial was reduced, when the preceding trial was an incongruent trial which caused strong ACC activation. As expected, after incongruent trials, the dorsolateral PFC was highly activated, thus confirming its role in conflict adaptation. 
Beside these effects of reactive control, other studies looked at the impact of cues which were predictive of the upcoming conflict (e.g., Sohn et al., 2007; Correa et al., 2008; Aarts and Roelofs, 2010). Sohn et al. (2007) presented input values in the form of two letters (e.g., B I) on which Boolean arithmetic tasks, according to different rules, had to be performed. All rules specified a relation between the input values (e.g., both I, one I, both not I, one not I) and an output value (e.g., I or B). Half of these rules implied low-conflict because they formed a positive relation between input and output (e.g., "if both input values are I, then the output is I"). The other half of the rules were high-conflicting because there was a negative relation between input and output (e.g., "if both input values are I, then the output is B"). Along with the input, an operator was presented which indicated which rule had to be performed on the input. On half of the trials, the authors cued the upcoming rule, by presenting the operator $9 \mathrm{~s}$ before the input. When no cue was presented, the ACC and lateral PFC were hardly activated. When a cue was presented, both areas were significantly more activated. Interestingly, the activation of the ACC and the lateral PFC was much higher when the cue predicted an upcoming high conflict trial, compared to an upcoming low-conflict trial. Although this seems to suggest that the ACC is not only involved in detecting response conflict, but also proactively signals that conflict is to be expected, a recent study observed no different ACC activity when comparing cues signaling upcoming congruent or incongruent trials with 75\% certainty (Aarts and Roelofs, 2010). Thus, although the ACC has been shown to be activated on conflict trials, its role in anticipatory behavior is currently under debate.

Although fMRI has a good spatial resolution, its temporal resolution is very limited. To circumvent this problem, EEG can be used to look at the time course of conflict situations. In EEG studies, activation of the ACC is believed to be expressed in the N2 (Ridderinkhof et al., 2004), a negative deflection in the EEG waveform with a fronto-central scalp distribution that peaks approximately 250-350 after stimulus presentation (Yeung and Cohen, 2006). Consistent with the role of the ACC in the conflict monitoring theory, the amplitude of this component is more negative on incompatible compared to compatible trials (Wendt and Luna-Rodriguez, 2009; Clayson and Larson, 2011; Purman et al., 2011). Another component typically associated with response conflict is the P3, a positive deflection in the EEG waveform with a central-parietal distribution occurring 350-500 ms after stimulus presentation (Clayson and Larson, 2011; Frühholz et al., 2011). The latency of this component is found to be prolonged for incongruent compared to congruent trials (Purman et al., 2011). Some suggest that this is because the $\mathrm{P} 3$ is an index of the stimulus evaluation (Kopp et al., 1996; Purman et al., 2011), and incongruent trials take more time to be evaluated. Others have suggested that the $\mathrm{P} 3$ represents response inhibition (Frühholz et al., 2011), since it is more positive on incongruent compared to congruent trials (Clayson and Larson, 2011; Frühholz et al., 2011). Evidence for this latter interpretation comes from a study which points to the inferior frontal cortex, an area associated with response inhibition, as the neural generator of the P3 (Nee et al., 2007). Clayson and Larson (2011) measured both the N2 and the P3 in an Eriksen flanker task and found evidence for conflict adaptation on these components. Mirroring the sequential modulation effect in the behavioral data, the difference in amplitude between an incongruent and a congruent trial was reduced when the previous trial was incongruent, compared to congruent. This was true for both the $\mathrm{N} 2$ and the P3.

In summary, a large body of fMRI data confirmed the role of the ACC in detecting response conflict, and the PFC in coping with this conflict. Also EEG studies support the notion that specific brain mechanisms respond to response conflict. To get more insights into the possibility of unconscious conflict to trigger control processes, these brain measures can also be used. If unconscious conflicting information is unable to activate the ACC and the PFC, this would suggest that conflict adaptation is a preserved area for consciousness.

\section{UNCONSCIOUS CONFLICT ADAPTATION IN THE BRAIN}

In a classical brain imaging study, Dehaene et al. (1998) found that even masked primes could activate the motor cortex. In the light of this observation, it should be a fruitful approach to look whether the brain areas activated by conscious conflict, are also activated when the conflict remains unconscious. Although an overwhelming number of studies focused on the role of the ACC in conflict situations, in the area of unconscious conflict adaptation this research is very scarce. In one isolated study, Dehaene et al. (2003) replicated their previous study (Dehaene et al., 1998) and looked whether trials containing response conflict (i.e., incongruent trials) activate the ACC. As expected, when primes were visible, large congruency effects were observed and the ACC was strongly activated when comparing conflict to no-conflict trials. Interestingly, when primes were masked, no differential ACC activity was observed when comparing both trial types, despite the presence of a significant congruency effect. Thus, although the unconscious irrelevant information hampered performance, its conflict with the relevant information did not trigger the ACC. In contrast, a recent study observed activation of the caudal ACC when comparing unconscious conflict with no-conflict trials (Ursu et al., 2009). In this experiment subjects had to respond to the position of consecutively presented faces (primary task), while remembering which faces were presented (secondary task). Unknown to participants, the position of each face predicted the position of the next face with $70 \%$ accuracy. Subjects implicitly learned this rule, as evidenced by faster reaction times on predicted locations, but never became aware of this contingency because the distraction task was too demanding. On the $30 \%$ of trials where the position of the face was not predicted, the implicitly learned sequence was violated, and although subjects never consciously noticed this, the caudal ACC was nevertheless activated during this conflict.

These contradicting results seem to suggest that unconscious conflicting information can activate conflict sensitive brain regions (Ursu et al., 2009), but only when the information itself was experienced consciously (Dehaene et al., 2003). However, an alternative explanation can account for this dissociation. In the study of Dehaene et al. the congruency effect in the masked condition was, although significant, twice as small as in the visible condition. So it is possible that these authors observed ACC activation only for conscious conflict, because the conflict conveyed by the masked primes was not sufficiently strong (Mayr, 2004). Whenever 
conflicting information has a larger impact in the conscious condition, as evidenced by larger congruency effects, this alternative cannot be ruled out.

Other studies have looked at the potential role of the pre-SMA in dealing with unconscious conflict. In a priming study, Wolbers et al. (2006) manipulated unconscious conflict frequency blockwise. They observed a tight coupling of the pre-SMA with the lateral occipital complex and the putamen, when comparing a block with $80 \%$ incongruent trials to a block with $80 \%$ congruent trials. Likewise, in another priming study, it was shown that the size of the congruency effect was negatively correlated with the pre-SMA gray-matter density, independent of the prime visibility (van Gaal et al., 2010d). Unfortunately, prime visibility of the masked primes in both studies was far above chance-level, so it is questionable whether these results tell us something about unconscious conflict.

In summary, although a few studies addressed the brain mechanisms responsible for resolving conflict which remains unconscious, there is an important gap in the literature on this topic. So in order to learn more about the mechanisms of unconscious conflict adaptation, more brain imaging research is necessary.

\section{GENERAL DISCUSSION}

In this review we discussed studies looking at the (im)possibility of conflict which remains unconscious, to have an influence on behavior. Answering this question will help us to unravel the purpose of consciousness. Much research was stimulated by the work of Dehaene and Naccache (2001), who speculated that consciousness is a prerequisite for top-down cognitive control. Only one single study convincingly showed trial-by-trial adaptation to unconscious conflict. (van Gaal et al., 2010b). All other studies indicate that modification of behavior on a trial-by-trial basis, depending on the conflict of the previous trial, is only possible when this conflict was experienced consciously. Evidence for blockwise adaptation to conflict, is much more ambiguous. Although some studies presented qualitative differences between conscious and unconscious conditions (e.g., Merikle and Joordens, 1997), there is much evidence that subjects can adapt to unconscious statistical regularities. However, it is currently debated whether this truly reflects adaptation to unconscious conflict (e.g., Bodner and Masson, 2001, 2004) or whether it is actually an adaptation to information which somehow reached consciousness (e.g., Jaskowski et al., 2003; Van den Bussche and Reynvoet, 2008). To date, only one recent study presented evidence that unconscious stimuli can create an unconscious context, while excluding meta-cognitive explanations (Van Opstal et al., 2011). An alternative to circumvent problems of behavioral studies is to look at brain measures of unconscious conflict. Unfortunately, although a large body of research explored the brain mechanisms involved in conscious conflict (Botvinick et al., 2004), this type of research is virtually lacking in the field of unconscious conflict adaptation.

In the remainder of this review we will discuss the conclusiveness of existing studies regarding these questions. We will look at strengths and weaknesses of all studies and give some suggestions how future research can tackle questions which research hitherto failed to answer conclusively.

\section{ARE WE LOOKING AT COGNITIVE CONTROL?}

An important point in the research on unconscious conflict adaptation is that we should be cautious about the origin of an effect. We should not be satisfied showing that a result can also be obtained when the conflicting information is masked, but also take into account the uncertainties this area faces. Namely, we should be sure that the behavior under investigation is an expression of cognitive control at all. For both issues discussed here, trial-by-trial and blockwise adaptation to response conflict, alternative explanations in terms of low-level learning have been proposed (Logan, 1988; Hommel, 1998). Hommel (1998) claimed that trial-by-trial adaptations could be perfectly explained without assuming any control process. He argued that trials are responded to fastest when the stimulus and response features are exact repetitions or complete alternations from these features on the previous trial. If only some features, but not all, overlap with the previous trial, this will create interference and slow down responses. This low-level learning mechanism can completely explain sequential modulations, without assuming cognitive control processes (e.g., Mayr et al., 2003; Hommel et al., 2004; Nieuwenhuis et al., 2006; Mayr and Awh, 2009). Likewise, blockwise adaptation to response conflict can also be explained by low-level learning mechanisms. When $80 \%$ of the trials are congruent, we have also practiced these trial types more often. It is well known that we become faster with more practice (Logan, 1988), so the effect can be perfectly explained, without assuming any influence of top-down cognitive control. It can even be argued that blockwise adaptation to response conflict is actually an expression of trial-by-trial adaptation (e.g., Kinoshita et al., 2011). If a block consists of $80 \%$ incongruent trials, the previous trial will be by definition incongruent on $80 \%$ of the trials. The cognitive system will react to this by reducing the influence of the irrelevant information (Gratton et al., 1992), which will reduce the congruency effect on $80 \%$ of the current trials. On average this will cause small congruency effects, which can be misleadingly taken as evidence for blockwise adaptation to conflict. Although there is compelling evidence that trial-by-trial adaptations (e.g., Ullsperger et al., 2005; Kunde and Wühr, 2006; Notebaert and Verguts, 2007), and blockwise adaptation (Tzelgov et al., 1992) cannot entirely be explained by these accounts, studies who try to show that these effects can also occur when conflict remains unconscious, should take these explanations into consideration and try to rule them out. For example, when manipulating conflict frequency blockwise, with only a limited number of stimuli (e.g., Klapp, 2007) reaction times are always confounded with the frequency with which certain prime-target combinations (i.e., congruent or incongruent) are exposed. To rule out this low-level learning mechanism, the stimulus set can be enlarged, so that all primes and targets occur equally often (Bodner and Masson, 2001; Egner and Hirsch, 2005; Crump and Milliken, 2009). Likewise, as long as two consecutive trials with the same congruency are complete repetitions or complete alternations, and two consecutive trials with a different congruency always partial repetitions, a feature explanation can always explain trial-by-trial adaptations. To rule out this explanation, more than two response options (Kunde and Wühr, 2006; Lamers and Roelofs, 2011) or more than two stimulus dimensions (Kunde and Wühr, 2006) can be used, so that all consecutive trials are complete alternations. 


\section{ARE WE COMPARING CONDITIONS BASED ON A DIFFERENCE IN CONSCIOUSNESS?}

To provide conclusive evidence that adaptation to response conflict cannot be initiated by unconscious stimuli, we have to ascertain that the only difference between the conscious and the unconscious condition is solely consciousness. In the conscious condition subjects need to be aware of the conflict and in the unconscious condition they must not be aware of the conflict, not on any level (e.g., Jaskowski et al., 2003; Van den Bussche et al., 2008). Although this seems a trivial requirement, existing paradigms do not seem to be able to satisfy this criterion. For example, Kunde (2003) and van Gaal et al. (2010b) varied the SOA to create a conscious and an unconscious condition. Frings and Wentura (2008) matched the SOA between both conditions, but failed to keep the prime duration constant. Even when prime duration and SOA are perfectly matched between both conditions (e.g., Ansorge et al., 2011), the absence of unconscious sequential modulations can still be explained by other accounts. Primes are typically followed by a maskin the unconscious condition and by a blank in the conscious condition. Because this mask rapidly destroys the presented image, this also impedes prime processing in this condition. As a consequence, the signal strength of the prime is much stronger in the unmasked condition (Lau, 2009; Francken et al., 2011), and these primes had more possibility to activate conflict sensitive regions (Dehaene et al., 2003), and influence behavior on the subsequent trial. This difference in signal strength is typically expressed in significantly smaller (e.g., Greenwald et al., 1996; Dehaene et al., 2003; Kunde, 2003; van Gaal et al., 2010b), or at least numerically smaller (e.g., Frings and Wentura, 2008; Ansorge et al., 2011) congruency effects in the unconscious condition. To deal with this problem, Francken et al. (2011) proposed to match for these low-level differences in signal strength by masking all primes equally long. To create conscious and unconscious conditions they used effective (i.e., meta-contrast masking for low-visible trials) and ineffective (i.e., pseudomasking for high-visible trials) masks. They observed identical priming effects in both conditions. However, although the authors showed that the priming effect for high and low visible primes is identical when the signal strength is matched, the question remains whether this is also the case with a truly unconscious condition. Nevertheless, this is an important first step, because only if also the signal strength is matched between both conditions, more firm conclusions can be drawn about unconscious trial-by-trial adaptation.

\section{ARE UNCONSCIOUS PRIMES UNOUESTIONABLY UNCONSCIOUS?}

As a final remark we want to discuss measures of prime visibility. If we want to be sure a prime was truly unconscious, we

\section{REFERENCES}

Aarts, E., and Roelofs, A. (2010). Attentional control in anterior cingulate cortex based on probabilistic cueing. J. Cogn. Neurosci. 23, 716-727.

Akçay, C., and Hazeltine, E. (2008). Conflict adaptation depends on task structure. J. Exp. Psychol. Hum. Percept. Perform. 34, 958-973.
Ansorge, U., Fuchs, I., Khalid, S., and Kunde, W. (2011). No conflict control in the absence of awareness. Psychol. Res. 75, 351-365.

Bodner, G. E., and Dypvik, A. T. (2005). Masked priming of number judgments depends on prime validity and task. Mem. Cognit. 33, 29-47.

have to provide strong evidence to support this. Again, although this seems a trivial requirement, many studies fail to satisfy this point. This ranges from no detection task at all (Merikle and Joordens, 1997; Bodner and Masson, 2001, 2004; Ortells et al., 2006), up to the absence of adequate follow-up analyzes when the detection task is above chance-level (Ortells et al., 2003; Bodner and Dypvik, 2005; Klapp, 2007). Additional analyses showing no correlation between our detection measure and our priming effect, a significant intercept showing priming at zero visibility, or the presence of the effect in a subgroup with zero visibility, can give us more confidence about the unconscious nature of the primes. Although the introduction of post detection tasks was a major progress in research on subliminal processing (Kouider and Dehaene, 2007), this measure does not suffice in research on unconscious conflict adaptation. This is because unconscious sequential modulations can be caused by trial-bytrial adaptations to the difficulty of the previous trial rather than to the conflict. Likewise, blockwise adaptation to unconscious conflict can be caused by adaptation to the conscious difficulty of a block. Therefore it is necessary to collect prime awareness data on every trial, to control for these possibilities. A disadvantage of this measure is that these long inter-trial interruptions possibly wipe out short-lived unconscious traces, which will impede trial-by-trial adaptation. Nevertheless, without this information we cannot dissociate between truly unconscious adaptation, and adaptation to information which somehow reached consciousness (Jaskowski et al., 2003; Van den Bussche et al., 2008).

\section{CONCLUSION}

In this review we gave a clear overview of research looking at the possibility of unconscious information to trigger adaptation behavior. Dehaene and Naccache (2001) speculated that top-down cognitive control is one class of behavior which can only be initiated consciously. Although there is some evidence that adaptation to unconscious conflict both on a trial-by-trial and blockwise basis is possible, almost all evidence suffers from serious methodological and theoretical problems, which hampers progression in this important field of research. In future research, it should be further clarified to which extent unconscious stimuli are able to trigger conflict adaptation, while avoiding the problems discussed in this review. This will help us to further elucidate the purpose of consciousness.

\section{ACKNOWLEDGMENTS}

We would like to thank Tom Verguts for useful comments on an earlier draft of this article.

Bodner, G. E., and Masson, M. E. J. (2001). Prime validity affects masked repetition priming: evidence for an episodic resource account of priming. J. Mem. Lang. 45, 616-647.

Bodner, G. E., and Masson, M. E. J. (2004). Beyond binary judgments: prime validity modulates masked repetition priming in the naming task. Mem. Cognit. 32, 1-11.
Bodner, G. E., Masson, M. E. J., and Richard, N. T. (2006). Repetition proportion biases masked priming of lexical decisions. Mem. Cognit. 34, 1298-1311.

Bodner, G. E., and Mulji, R. (2010). Prime proportion affects masked priming of fixed and free-choice responses. Exp. Psychol. 57, 360-366. 
Botvinick, M. M. (2007). Conflict monitoring and decision making: reconciling two perspectives on anterior cingulate function. Cogn. Affect. Behav. Neurosci. 7, 356-366.

Botvinick, M. M., Braver, T. S., Barch, D. M., Carter, C. S., and Cohen, J. D. (2001). Conflict monitoring and cognitive control. Psychol. Rev. 108, 624-652.

Botvinick, M. M., Cohen, J. D., and Carter, C. S. (2004). Conflict monitoring and anteriorcingulate cortex: an update. Trends Cogn. Sci. 8 , 539-546.

Clayson, P. E., and Larson, M. J. (2011). Conflict adaptation and sequential effects: support for the conflict monitoring theory. Neuropsychologia 49, 1953-1961.

Cohen, M. X., van Gaal, S., Ridderinkhof, K. R., and Lamme, V. A. F. (2009). Unconscious errors enhance prefrontal-occipital oscillatory synchrony. Front. Hum. Neurosci. 3:54. doi:10.3389/neuro.09.054.2009

Corallo, G., Sackur, J., Dehaene, S., and Sigman, M. (2008). Limits on introspection: distorted subjective time during the dual-task bottleneck. Psychol. Sci. 19, 1110-1117.

Correa, A., Rao, A., and Nobre, A. C. (2008). Anticipating conflict facilitates controlled stimulus-response selection. J. Cogn. Neurosci. 21, 1461-1472.

Crump, M. J. C., Gong, Z., and Milliken, B. (2006). The context-specific proportion congruent Stroop effect: location as a contextual cue. Psychon. Bull. Rev. 13, 316-321.

Crump, M. J. C., and Milliken, B. (2009). The flexibility of context-specific control: evidence for context-driven generalization of item-specific control settings. Q. J. Exp. Psychol. 62, 1523-1532.

Damian, M. (2001). Congruity effects evoked by subliminally presented primes: automaticity rather than semantic processing. J. Exp. Psychol. Hum. Percept. Perform. 27, 154-165.

Daza, M. T., Ortells, J. J., and Fox, E. (2002). Perception without awareness: further evidence from a Stroop priming task. Percept. Psychophys. 64, 1316-1324.

Daza, M. T., Ortells, J. J., and Noguera, C. (2007). Negative semantic priming from consciously vs. unconsciously perceived single words. Psicologica 28, 105-127.

De Pisapia, N., Turatto, M., Lin, P., Jovicich, J., and Caramazza, A. (in press). Unconscious priming instructions modulate activity in default and executive networks of the human brain. Cereb. Cortex. doi:10.1093/cercor/bhr146

Dehaene, S., Artiges, E., Naccache, L., Martelli, C., Viard, A., Schürhoff, F., Recasens, C., Martinot, M. L. P., Leboyer, M., and Martinot, J. L. (2003). Conscious and subliminal conflicts in normal subjects and patients with schizophrenia: the role of the anterior cingulate. Proc. Natl. Acad. Sci. U.S.A. 100, 13722-13727

Dehaene, S., and Naccache, L. (2001). Towards a cognitive neuroscience of consciousness: basic evidence and a workspace framework. Cognition 79, 1-37.

Dehaene, S., Naccache, L., Le Clec'H, G., Koechlin, E., Mueller, M., DehaeneLambertz, G., van de Moortele, P. F., and Le Bihan, D. (1998). Imaging unconscious semantic priming. Nature 395, 597-600.

Egner, T. (2008). Multiple conflictdriven control mechanisms in the human brain. Trends Cogn. Sci. 12, 374-380.

Egner, T., and Hirsch, J. (2005). Cognitive control mechanisms resolve conflict through cortical amplification of task-relevant information. Nat. Neurosci. 8, 1784-1790.

Eimer, M., and Schlaghecken, F. (1998). Effects of masked stimuli on motor activation: behavioral and electrophysiological evidence. J. Exp. Psychol. Hum. Percept. Perform. 24, 1737-1747.

Eriksen, B. A., and Eriksen, C. W. (1974). Effects of noise letters upon the identification of a target letter in a nonsearch task. Percept. Psychophys. 16, 143-149.

Eriksen, C. W. (1960). Discrimination and learning without awareness: a methodological survey and evaluation. Psychol. Rev. 67, 279-300.

Evett, L. J., and Humphreys, G. W. (1981). The use of abstract graphemic information in lexical access. Q. J. Exp. Psychol. 33, 325-350.

Forster, K. I., and Davis, C. (1984). Repetition priming and frequency attenuation in lexical access. J. Exp. Psychol. Learn. Mem. Cogn. 10, 680-698.

Francken, J. C., van Gaal, S., and de Lange, F. P. (2011). Immediate and long-term priming effects are independent of prime awareness. Conscious. Cogn. 20, 1793-1800.

Frings, C., and Wentura, D. (2008). Trial-by-trial effects in the affective priming paradigm. Acta Psychol. 128, 318-323.

Frühholz, S., Godde, B., Finke, M., and Herrmann, M. J. (2011). Spatio-temporal brain dynamics in a combined stimulus-stimulus and stimulus-response conflict task. Neuroimage 54, 622-634.

Gratton, G., Coles, M. G. H., and Donchin, E. (1992). Optimizing the use of information: strategic control of activation of responses. J. Exp. Psychol. Gen. 121, 480-506.

Greenwald, A. G., Draine, S. C., and Abrams, R. L. (1996). Three cognitive markers of unconscious semantic activation. Science 273 1699-1702.

Heinemann, A., Kunde, W., and Kiesel, A. (2009). Context-specific primecongruency effects: on the role conscious stimulus representations for cognitive control. Conscious. Cogn. 18, 966-976.

Hester, R., Foxe, J. J., Molholm, S., Shpaner, M., and Garavan, H. (2005). Neural mechanisms involved in error processing: a comparison of errors made with and without awareness. Neuroimage 27 602-608.

Holender, D. (1986). Semantic activation without conscious identification in dichotic listening, parafoveal vision, and visual masking: a survey and appraisal. Behav. Brain Sci. 9, $1-23$.

Hommel, B. (1998). Event files: evidence for automatic integration of stimulus-response episodes. Vis. Cogn. 5, 183-216.

Hommel, B., Proctor, R. W., and Vu, K. P. (2004). A feature-integration account of sequential effects in the Simon task. Psychol. Res. 68, 1-17.

Jaskowski, P. (2008). Conscious contributions to subliminal priming. Conscious. Cogn. 17, 72-83.

Jaskowski, P., Skalska, B., and andVerleger, R. (2003). How the self controls its "automatic pilot" when processing subliminal information. J. Cogn. Neurosci. 15, 911-920.

Kerns, J. G., Cohen, J. D., MacDonald, A. W. III, Cho, R. Y., Stenger, V. A., and Carter, C. S. (2004). Anterior cingulate conflict monitoring and adjustments in control. Science 303, 1023-1026.

Kinoshita, S., Forster, K. I., and Mozer, M. I. (2008). Unconscious cognition is not that smart: modulation of masked repetition priming effect in the word naming task. Cognition $107,623-649$.

Kinoshita, S., and Hunt, L. (2008). RT distribution analysis of category congruence effects with masked primes. Mem. Cognit. 36, 1324-1334.

Kinoshita, S., Mozer, M. I., and Forster K. I. (2011). Dynamic adaptation to history of trial difficulty explains the effect of congruency proportion on masked priming. J. Exp. Psychol. Gen. 140, 622-636.

Klapp, S. T. (2007). Nonconscious control mimics a purposeful strategy: strength of Stroop-like interference is automatically modulated by proportion of compatible trials. J. Exp. Psychol. Hum. Percept. Perform. 33, 1366-1376.

Kopp, B., Rist, F., and Mattler, U. (1996). N200 in the flankers task as a neurobehavioral tool for investigating executive control. Psychophysiology 33, 282-294.

Kouider, S., and Dehaene, S. (2007). Levels of processing during nonconscious perception: a critical review of visual masking. Philos. Trans. R. Soc. Lond. B Biol. Sci. 362, 857-875.

Kunde, W. (2003). Sequential modulations of stimulus-response correspondence effects depend on awareness of response conflict. Psychon. Bull. Rev. 10, 198-205.

Kunde, W., and Wühr, P. (2006). Sequential modulations of correspondence effects across spatial dimensions and tasks. Mem. Cognit. 34, 356-367.

Kunde, W., Kiesel, A., and Hoffmann, J. (2003). Conscious control over the content of unconscious cognition. Cognition 88, 223-242.

Lamers, M. J. M., and Roelofs, A. (2011). Attentional control adjustments in Eriksen and Stroop task performance can be independent of response conflict. Q. J. Exp. Psychol. 64, 1056-1081.

Lau, H. C. (2009). "Volition and the functions of consciousness," in The Cognitive Neurosciences IV, ed. M. Gazzaniga (Boston: MIT Press), 153-169.

Lau, H. C., and Passingham, R. E. (2007). Unconscious activation of the cognitive control system in the human prefrontal cortex. J. Neurosci. 27, 5805-5811.

Libet, B. (1985). Unconscious cerebral initiative and the role of conscious will in voluntary action. Behav. Brain Sci. 8, 529-539.

Logan, G. D. (1988). Toward an instance theory of automatization. Psychol. Rev. 95, 492-527.

Logan, G. D., and Zbrodoff, J. N. (1979). When it helps to be misled: facilitative effects of increasing the frequency of conflicting stimuli in a Stroop-like task. Mem. Cognit. 7 , 166-174.

MacLeod, C. M., and MacDonald, P. A. (2000). Interdimensional interference in the Stroop effect: uncovering the cognitive and neural anatomy 
of attention. Trends Cogn. Sci. 4, 383-391.

Martens, U., Ansorge, U., and Kiefer, M. (2011). Controlling the unconscious: attentional task sets modulate subliminal semantic and visuomotor processes differentially. Psychol. Sci. 22, 282-291.

Marti, S., Sackur, J., Sigman, M., and Dehaene, S. (2010). Mapping introspection's blind spot: reconstruction of dual-task phenomenology using quantified introspection. Cognition 115, 303-313.

Mayr, U. (2004). Conflict, consciousness, and control. Trends Cogn. Sci. 8, 145-148.

Mayr, U., and Awh, E. (2009). The elusive link between conflict and conflict adaptation. Psychol. Res. 73, 794-802.

Mayr, U., Awh, E., and Laurey, P. (2003). Conflict adaptation effects in the absence of executive control. Nat. Neurosci. 6, 450-452.

Merikle, P. M., and Joordens, S. (1997). Parallels between perception without attention and perception without awareness. Conscious. Cogn. 6 , 219-236.

Naccache, L., Blandin, E., and Dehaene, S. (2002). Unconscious masked priming depends on temporal attention. Psychol. Sci. 13, 416-424.

Nee, D. E., Wager, T. D., and Jonides, J. (2007). Interference resolution: insights from a meta-analysis of neuroimaging tasks. Cogn. Affect. Behav. Neurosci. 7, 1-17.

Nieuwenhuis, S., Stins, J. F., Posthuma, D., Polderman, T. J. C., Boomsma, D. I., and De Geus, E. J. (2006). Accounting for sequential trial effects in the flanker task: conflict adaptation or associative priming? Mem. Cognit. 34, 1260-1272.

Norris, D., and Kinoshita, S. (2008). Perception as evidence accumulation and Bayesian inference: insights from masked priming. J. Exp. Psychol. Gen. 17, 434-455.

Notebaert, W., Gevers, W., Verbruggen, F., and Liefooghe, B. (2006). Topdown and bottom-up sequential modulations of congruency effects. Psychon. Bull. Rev. 13, 112-117.

Notebaert, W., and Verguts, T. (2007). Dissociating conflict adaptation from feature integration: a multiple regression approach. J. Exp. Psychol. Hum. Percept. Perform. 33, 1256-1260.

Ortells, J. J., Daza, M. T., and Fox, E. (2003). Semantic activation in the absence of perceptual awareness. Percept. Psychophys. 65, 1307-1317.

Ortells, J. J., Vellido, C., Daza, M. T., and Noguera, C. (2006). Semantic priming effects from conscious and unconsciously perceived words. Psicologica 27, 225-242.

Pratkanis, A. R. (1992). The cargo-cult science of subliminal persuasion. Skeptical Inquirer 16, 260-272.

Purman, S., Badde, S., Luna-Rodriguez, A., and Wendt, M. (2011). Adaptation to frequent conflict in the Eriksen flanker task: an ERP study. J. Psychophysiol. 25, 50-59.

Reuss, H., Kiesel, A., Kunde, W., and Hommel, B. (2011). Unconscious activation of task sets. Conscious. Cogn. 20, 556-567.

Ridderinkhof, K. R. (2002). Micro- and macro-adjustments of task set: activation and suppression in conflict tasks. Psychol. Res. 66, 312-323.

Ridderinkhof, K. R., Ullsperger, M., Crone, E. A., and Nieuwenhuis, S. (2004). The role of the medial frontal cortex in cognitive control. Science 306, 443-447.

Sohn, M. H., Albert, M. V., Jung, K., Carter, C. S., and Anderson, J. R. (2007). Anticipation of conlict monitoring in the anterior cingulate cortex and the prefrontal cortex. Proc. Natl. Acad. Sci. U.S.A. 104, 10330-10334.

Soon, C. S., Brass, M., Heinze, H. J., and Haynes, J. D. (2008). Unconscious determinants of free decisions in the human brain. Nat. Neurosci. 11, 543-545.

Stürmer, B., Leuthold, H., Soetens, E., Schröter, H., and andSommer, W. (2002). Control over locationbased response activation in the Simon task: behavioral and electrophysiological evidence. J. Exp. Psychol. Hum. Percept. Perform. 28, 1345-1363.

Tzelgov, J., Henik, A., and Berger, J. (1992). Controlling Stroop effects by manipulating expectations for color words. Mem. Cognit. 20, 727-735.

Ullsperger, M., Bylsma, L. M., and Botvinick, M. M. (2005). The conflict adaptation effect: it's not just priming. Cogn. Affect. Behav. Neurosci. 5, 476-472.

Ursu, S., Clark, K. A., Aizenstein, H. J, Stenger, V. A., and Carter, C. S. (2009). Conflict-related activity in the caudal anterior cingulate cortex in the absence of awareness. Biol. Psychol. 80, 279-286.
Van den Bussche, E., Hughes, G. Van Humbeeck, N., and Reynvoet, B. (2010). The relation between consciousness and attention: an empirical study using the priming paradigm. Conscious. Cogn. 19 86-97.

Van den Bussche, E., Notebaert, K., and Reynvoet, B. (2009a). Masked primes can be genuinely semantically processed a picture prime study. Exp. Psychol. 56, 295-300.

Van den Bussche, E., Van den Noortgate, W., and Reynvoet, B. (2009b). Mechanisms of masked priming: a meta-analysis. Psychol. Bull. 135 , 458-477.

Van den Bussche, E., and Reynvoet, B. (2008). The asymmetry between top-down effects and unconscious cognition: additional issues. Conscious. Cogn. 17, 1361-1363.

Van den Bussche, E., Segers, G., and Reynvoet, B. (2008). Conscious and unconscious proportion effects in masked priming. Conscious. Cogn. 17, 1345-1358.

Van Opstal, F., Calderon, C. B., Gevers, W., and Verguts, T. (2011). Setting the stage subliminally: unconscious context effects. Conscious. Cogn. 20 , 1860-1864.

Van Opstal, F., Gevers, W., Osman, M., and Verguts, T. (2010). Unconscious task application. Conscious. Cogn. 19, 999-1006

van Gaal, S., Lamme, V. A. F. Fahrenfort, J. J., and Ridderinkhof, K. R. (2010a). Dissociable brain mechanisms underlying the conscious and unconscious control of behavior. J. Cogn. Neurosci. 23 , 91-105.

van Gaal, S., Lamme, V. A. F., and Ridderinkhof, K. R. (2010b). Unconsciously triggered conflict adaptation. PLOS ONE 5, el1508. doi:10.1371/journal.pone.0011508

van Gaal, S., Riddenrinkhof, K. R., Scholte, H. S., and Lamme, V. A. F. (2010c). Unconscious activation of the prefrontal no-go network. $J$. Neurosci. 30, 4143-4150.

van Gaal, S., Scholte, S., Lamme, V. A. F., Fahrenfort, J. J., and Ridderinkhof, K. R. (2010d). Pre-SMA gray-matter density predicts individual differences in action selection in the face of conscious and unconscious response conflict. J. Cogn Neurosci. 23, 382-390.

Verguts, T., Notebaert, W., Kunde, W. and Wühr, P. (2011). Post-conflict slowing: cognitive adaptation after conflict processing. Psychon. Bull. Rev. 18, 76-82.

Vorberg, D., Mattler, U., Heinecke, A., Schmidt, T., and Schwarzbach J. (2003). Different time courses for visual perception and action priming. Proc. Natl. Acad. Sci. U.S.A 100, 6275-6280.

Wegner, D. M. (2002). The Illusion of Conscious Will. Cambridge: MIT Press.

Wendt, M., and Luna-Rodriguez, A. (2009). Conflict-frequency affects flanker interference: role of stimulus-ensemble specific practice and flanker-response contingencies. Exp. Psychol. 56, 206-217.

Wenke, D., Fleming, S. M., and Haggard, P. (2010). Subliminal priming of actions influences sense of control over effects of action. Cognition 115, 26-38.

Wolbers, T., Schoell, E. D., Verleger, R., Kraft, S., McNamara, A., Jaskowski P., and Büchel, C. (2006). Changes in connectivity profiles as a mechanism for strategic control over interfering subliminal information. Cereb. Cortex 16, 857-864.

Wühr, P., and Frings, C. (2008). A case for inhibition: visual attention suppresses the processing of irrelevant objects. J. Exp. Psychol. Gen. 137, 116-130.

Yeung, N., and Cohen, J. D. (2006). The impact of cognitive deficits on conflict monitoring predictable dissociations between the error-related negativity and N2. Psychol. Sci. 17, 164-171.

Conflict of Interest Statement: The authors declare that the research was conducted in the absence of any commercial or financial relationships that could be construed as a potential conflict of interest.

Received: 15 December 2011; accepted: 16 January 2012; published online: 03 February 2012.

Citation: Desender $K$ and Van den Bussche E (2012) Is consciousness necessary for conflict adaptation? A state of the art. Front. Hum. Neurosci. 6:3. doi: 10.3389/fnhum.2012.00003 Copyright (c) 2012 Desender and Van den Bussche. This is an open-access article distributed under the terms of the Creative Commons Attribution Non Commercial License, which permits noncommercial use, distribution, and reproduction in other forums, provided the original authors and source are credited. 